\title{
Artificial Recurrent Neural Network Architecture in Customer Consumption Prediction for Business Development
}

\author{
Dr. P. Karuppusamy \\ Professor, Department of EEE, \\ Shree Venkateshwara Hi-Tech Engineering College, \\ Erode, India \\ E-mail: pkarrupusamyphd@gmail.com
}

\begin{abstract}
The customer consumption pattern prediction has become one of a significant role in developing the business and taking it to a competitive edge. For forecasting the behaviors of the consumers the paper engages an artificial recurrent neural network architecture the long short-term memory an improvement of recurrent neural network. The mechanism laid out to predict the pattern of the consumption, uses the information's about the consumption of products based on the age and the gender. The information essential are extracted and described with the prefix-span procedure based association rule. Utilizing the information about the day to day products purchase pattern as input a frame work to predict the customer daily essentials was designed, the designed frame was capable enough to learn the dissimilarities across the predicted and the original miscalculation rates. The frame work devised was tested using real life applications and the results observed demonstrated that the proposed LSTM based prediction with the prefix span association rule to acquire the day today consumption details is compatible for forecasting the customer consumption over time accurately.
\end{abstract}

Keywords: Artificial Recurrent Neural Network, Deep Learning, Customer Consumption, Long Short Term Memory, Accuracy, Miscalculation Rate

\section{Introduction}

Conducting Statistical evaluation of the consumer's activities in purchasing their essential needs has become an important requisite for forecasting the developments in business and know the degree of marketing required. The capability of forecasting the customer demands accurately could be helpful in efficiently targeting the products, and achieving a considerable improvement in the overall business process

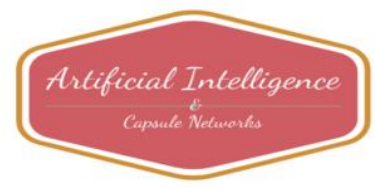


Journal of Artificial Intelligence and Capsule Networks (2020)

Vol.02/ No. 02

Pages: $111-120$

http://irojournals.com/aicn/

DOI: https://doi.org/10.36548/jaicn.2020.2.004

by the cost effective advertising approaches and as well as heightening the sales. Apart from the models developed using the logistic regression and the discriminant analysis in forecasting the consumer choices to enhance the performance the author Gan, Christopher et al [1] uses the artificial neural networks to devise a model predicting the consumer choices, by experimentally co-relating the forecasting potency of the PNNprobabilistic neural network and the other logistic models over the customer banking decision between the electronic and the non-electronic banking. As predicting the customer choice is very necessary the author Afolabi, Ibukun T., et al [2] has provided the systematic review based on the existing research on this topic. Comprehensive view of the consumer choices with the dataset about the relationship management and methods is delivered with the capability to sort out the limitations prevailing in the existing research methods on the subject matter and sort out the future research opportunities in the predicting the behavior of the customer. The chart in the figure. 1 is about the ratio of the components necessary in consumer relation management while predicting the choices of the customers.

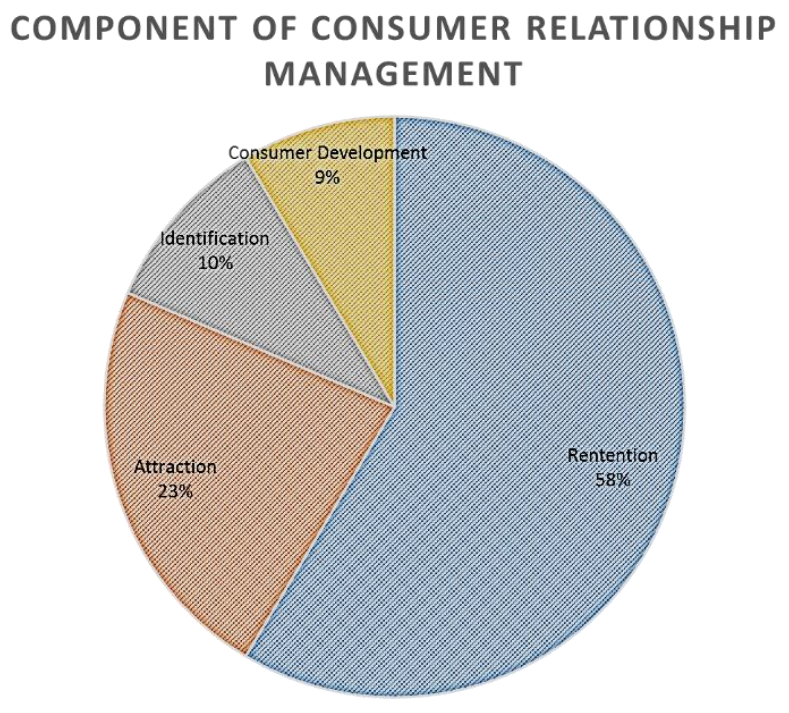

Figure.2 Components of Consumer Relationship Management [2]

The ultra-modern era of technology and the expectation of the marketing trends makes necessary for the observation of the customer activity in the world with the competitiveness and dynamic changes in the trends. The research put forth by the author Valecha et al [3] monitors the activities of the customer's behavior parameters and their willingness to purchase. The examination takes into consideration the environmental, organization and the individual and interpersonal aspects and forecast the behavior devising

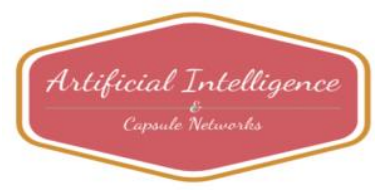


a random forest classifier model. Krebs et al [4] used the convolutional neural network in predicting the consumer activities from the wide range of dataset collected from the Facebook regarding the super market chains pages maintained. Urso et al [5] has put forth the demining strategies involved in predicting and classifying. The recurrent neural network relied methodologies are laid out using the past information as it is essential in predicting the consumption pattern of the human.

Although the past information's are considered in the RNN it still has few limitations such as "long term dependency problem" causing difficulties in predicting the details of the distant past. To resolve the problems arising in the RNN the Long short term memory the artificial recurrent neural network used in the field of deep learning is engaged. Unlike the state of art FNN (feed forward neural networks) the long short term has feedback connections and the capacity to compute a complete sequence of data, it well suited for un-segmented chores such as the handwriting recognition, speech recognition etc.

Moreover it is highly compatible for predicting the series of data based on time as there are unknown period lags in the important occurrence in a time series. It handles well the "vanishing gradient problem" that occurs while training the conventional recurrent neural network. "Relative insensitivity to gap length is an advantage of LSTM over the HMM-hidden markov model, RNN and the other sequence learning procedures involved in various applications.

So the proposed method to predict the consumption pattern of the consumers, devises a model using the LSTM to forecast the consumer behavior, the pattern of consumption is extracted using the prefix span based association rule analysis and the prediction is done based on the everyday learning about the behavior pattern.

The rest of the paper is arranged with the literature survey about the past works in section 2, the proposed approach in section 3, results and discussion in section 4 and the conclusion in section 5 .

\section{Literature Survey}

Sherstinsky, Alex yet al [6] has elaborated the basics of the recurrent neural networks and the long short term memory network. Staudemeyer,et al [7] presents the tutorial on the long short memory recurrent neural network Qing et al [8] presents the "Hourly day-ahead solar irradiance prediction using weather forecasts by LSTM."

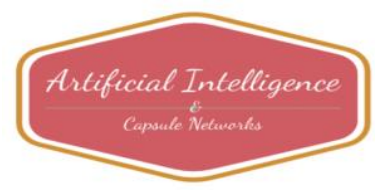


Journal of Artificial Intelligence and Capsule Networks (2020)

Vol.02/ No. 02

Pages: 111-120

http://irojournals.com/aicn/

DOI: https://doi.org/10.36548/jaicn.2020.2.004

Mane, et al [9] has utilized the prefix span based association rule analysis in the predicting the student admission, Raj, Jennifer S et al [10] elaborates the "A Comprehensive Survey on the Computational Intelligence Techniques and Its Applications." Joseph, S. Iwin et al [11] discusses the "Survey of data mining algorithms for intelligent computing system." J. Vijitha Ananthi et al [12] has devised a "Recurrent Neural Networks and Nonlinear Prediction in Support Vector Machines." Bashar, Abul et al [13] presents a "Survey on Evolving Deep Learning Neural Network Architectures."

\section{Proposed Prediction Strategy}

The architecture for predicting the consumer behavior is structured into four phases with as shown in the flow chart below in figure. 2 The raw data about the consumer consumption taken as input into the model is preprocessed by grouping the consumer based on their age as well as gender and filtering the unwanted information's other than the age, gender and their requirements. This done on the basis of statistical data of daily essential needs. The filtered data is further converted into a continuous data and subjected to the next phase that analyses the data sequence using the prefix span based association rules. The everyday details of the consumer behavior. To determine the main consumption model, the frequent consumption model is sorted out. This fedot the LSTM to learn and forecast the actual consumption details of the consumers. The difference between the original and the forecasted outcome is also learned to improve the accuracy in the prediction.

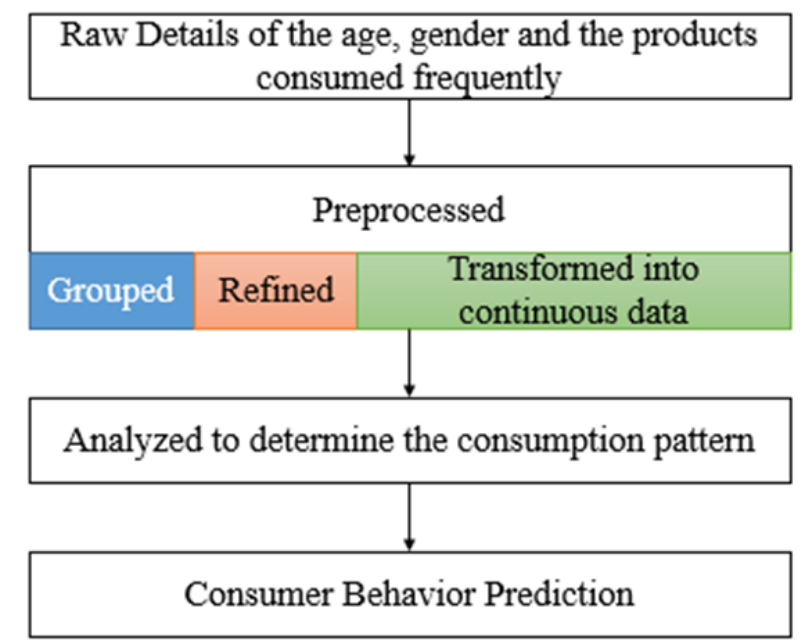

Figure.2 Proposed Flow Chart

114

ISSN: 2582-2012 (online)

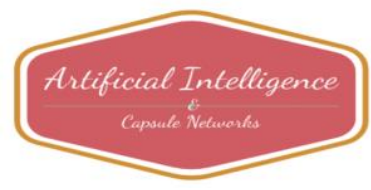


Journal of Artificial Intelligence and Capsule Networks (2020)

Vol.02/ No. 02

Pages: 111-120

http://irojournals.com/aicn/

DOI: https://doi.org/10.36548/jaicn.2020.2.004

The prefix span mines the sequential pattern by projection made on frequent prefix instead of projecting sequences database by determining the frequent occurrences of the subsequences. This causes minimization in the processing time completely enhancing the efficiency of the model. The result obtained is used as the input for predicting model that is the last stage of the process. The figure. 3 shows the long short term memory network's structure that is used in the prediction process.

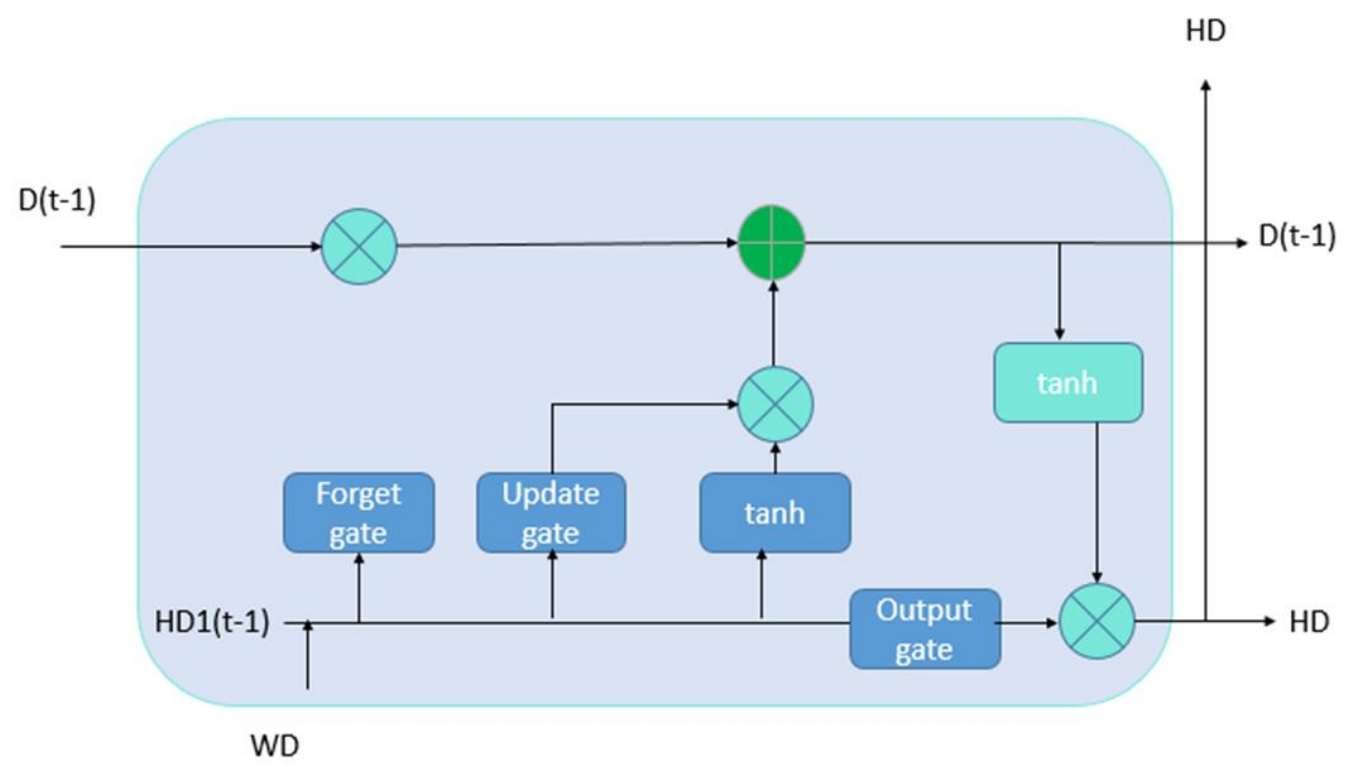

Figure.3 Cell Structure of LSTM

The fundamental operations of the long short term memory is to commune the outcomes based on the learning of the preceding state of the cells. From the figure .3 it is clear that the processing initially takes the sigmoid of the previous hidden state value as well as the input value from the forget gate and estimates the whether the data is to be preserved or not preserved across the ' 0 ' and the ' 1 '. In the next step an other sigmoid and the tanh is utilized as gate to estimate the whether the prevailing information is preserved in the input gate and it is updated or not. At last the sigmoid consider the value and the tanh estimates the outcome.

As the patter of consumption differs from person to person and from one day and other. The product consumption model of a week is computed based on the age, and the products purchased. With seventy

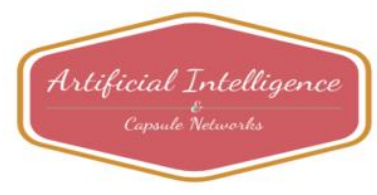


Journal of Artificial Intelligence and Capsule Networks (2020)

Vol.02/ No. 02

Pages: 111-120

http://irojournals.com/aicn/

DOI: https://doi.org/10.36548/jaicn.2020.2.004

nodes in the structure, for predicting the weekly consumption, the type of consumption is forecasted and the further learns by estimating the error rate in prediction from the actual.

\section{Results and Discussion}

The LSTM network is developed with the seventy nodes, one time stamp, the one input feature in the visible layer, 10 memory units in the hidden layer and one neural in the fully connected output layer with the default activation function is compiled with effective optimization algorithm along with the default settings and the mean squared error loss function. The system developed is trained with $80 \%$ the real time data set that are gathered and refined on the basis of daily routine consumption and the $20 \%$ of dataset are used in testing the model.

The results observed shows the accuracy of the models on training and predicting for 50 epochs by calculating the mean squared error, as shown in equation (1)

$$
M S E=\frac{1}{n} \sum_{i=1}^{n}\left(y_{i}-\breve{y}_{i}\right)^{\wedge} 2
$$

Where $y_{i}$ is the actual and the $\check{y}_{i}$ is the predicted value, by estimating the root mean square as shown in the equation (2)

$$
R M S E=\sqrt{M S E}
$$

And by finally determining the mean absolute error as shown in equation (3)

$$
M A P E=\frac{100}{n} \sum_{n=1}^{n}\left|\frac{A_{v}-F_{v}}{A_{v}}\right|
$$

Where $F_{v}=$ forecasted value $A_{t}=$ actual value. The figure .4 is the accuracy of the forecasting outcomes using the LSTM models this compared with the other model such as BPNN, KNN and the Extreme learning machine (ELM) the results shows that the accuracy of the proposed forecaster is better compared to the other methods.

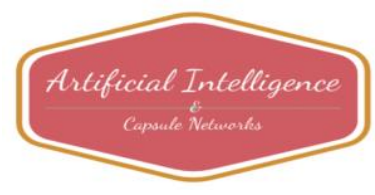


Journal of Artificial Intelligence and Capsule Networks (2020)

Vol.02/ No. 02

Pages: 111-120

http://irojournals.com/aicn/

DOI: https://doi.org/10.36548/jaicn.2020.2.004

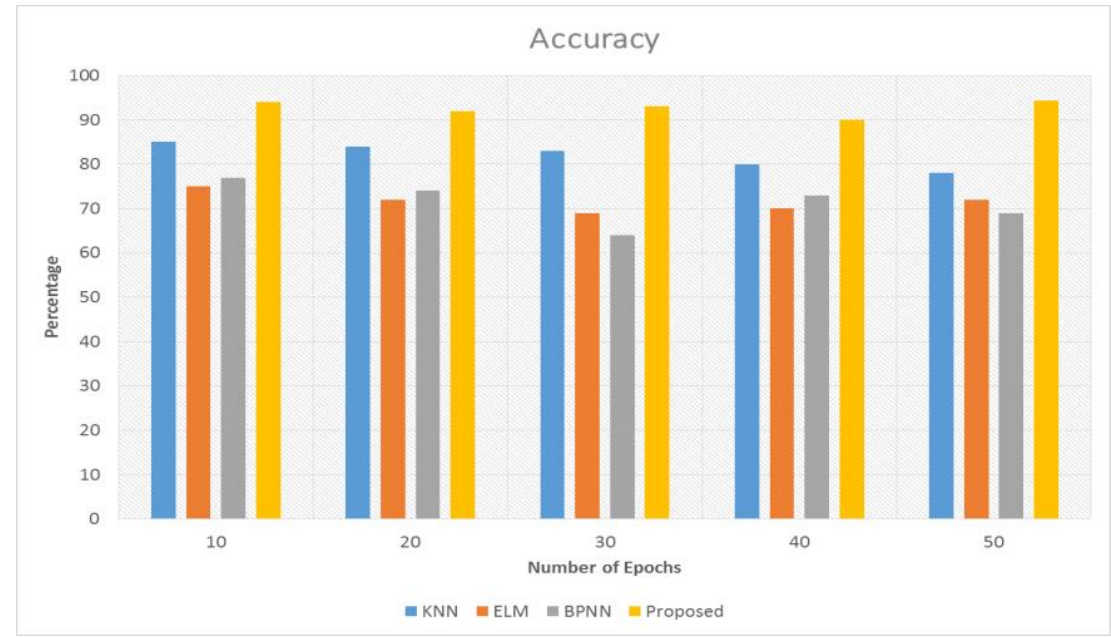

Figure. 4 Accuracy

Figure .5 depicts the error rate observed in every forecasting model based LSTM, KNN, BPNN, and ELM. The error rate observed further proves that the forecasting through LSTM is accurate compared to the other predicting models.

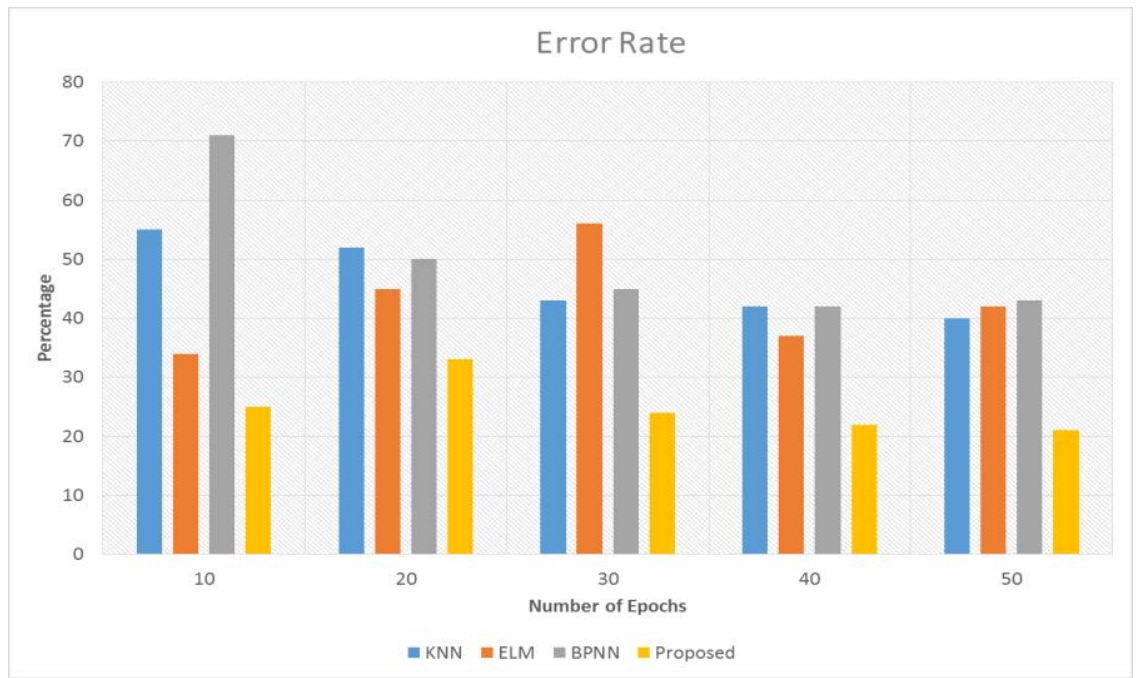

Figure .5 Error Rate

117

ISSN: 2582-2012 (online)

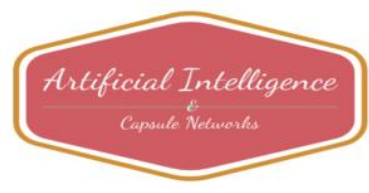


Journal of Artificial Intelligence and Capsule Networks (2020)

Vol.02/ No. 02

Pages: 111-120

http://irojournals.com/aicn/

DOI: https://doi.org/10.36548/jaicn.2020.2.004

The Table .1 below shows the Mean absolute error observed in the proposed and the other model along with the time consumed in analyzing the data and the memory used. The prefix span method used in the paper for analyzing and sequencing the data, based on the frequency of the prefix reduced the time and the memory consumed enhancing the efficiency of the proposed LSTM based predicting model. Thus proving the proposed method to be efficient than the other models compared.

\begin{tabular}{|c|c|c|c|c|c|c|c|c|c|c|c|c|c|c|c|c|c|c|c|c|}
\hline \multirow[t]{2}{*}{ Metrics } & \multicolumn{5}{|l|}{ KNN } & \multicolumn{5}{|l|}{ ELM } & \multicolumn{5}{|l|}{ BPNN } & \multicolumn{5}{|c|}{ Proposed } \\
\hline & $\begin{array}{l}10 \\
\text { Epochs }\end{array}$ & $\begin{array}{l}20 \\
\text { Epochs }\end{array}$ & $\begin{array}{l}30 \\
\text { Epochs }\end{array}$ & $\begin{array}{l}40 \\
\text { Epochs }\end{array}$ & $\begin{array}{l}50 \\
\text { Epochs }\end{array}$ & $\begin{array}{l}10 \\
\text { Epochs }\end{array}$ & $\begin{array}{l}20 \\
\text { Epochs }\end{array}$ & $\begin{array}{l}30 \\
\text { Epochs }\end{array}$ & $\begin{array}{l}40 \\
\text { Epochs }\end{array}$ & $\begin{array}{l}50 \\
\text { Epochs }\end{array}$ & $\begin{array}{l}10 \\
\text { Epochs }\end{array}$ & $\begin{array}{l}20 \\
\text { Epochs }\end{array}$ & $\begin{array}{l}30 \\
\text { Epochs }\end{array}$ & $\begin{array}{l}40 \\
\text { Epochs }\end{array}$ & $\begin{array}{l}50 \\
\text { Epochs }\end{array}$ & $\begin{array}{l}10 \\
\text { Epochs }\end{array}$ & $\begin{array}{l}20 \\
\text { Epochs }\end{array}$ & $\begin{array}{l}30 \\
\text { Epochs }\end{array}$ & $\begin{array}{l}40 \\
\text { Epochs }\end{array}$ & $\begin{array}{l}50 \\
\text { Epochs }\end{array}$ \\
\hline MAPE \% & 56 & 50 & 57 & 45 & 43 & 42 & 43 & 55 & 65 & 49 & 39 & 45 & 42 & 55 & 60 & 21 & 22 & 23 & 25 & 27 \\
\hline $\begin{array}{l}\text { Memory utilization } \\
\%\end{array}$ & 67 & 75 & 78 & 89 & 85 & 55 & 67 & 69 & 75 & 80 & 72 & 69 & 80 & 79 & 82 & 45 & 42 & 36 & 32 & 35 \\
\hline $\begin{array}{l}\text { Time Consumed } \\
\text { seconds }\end{array}$ & .045 & .055 & .057 & .062 & .064 & .063 & .045 & .058 & .057 & .034 & .059 & .065 & .056 & .045 & .57 & .034 & .026 & .021 & .020 & .198 \\
\hline
\end{tabular}

Table.1 MAPE, Time Consumption and Memory Utilization

\section{Conclusion}

The paper devises an artificial recurrent neural network (LSTM) based forecasting model to predict the customer consumption pattern to develop the business and the marketing strategies. The LSTM was utilized to subsidize the long term dependency problem found in the RNN and enhance the forecasting accuracy over time. The prefix span used in analyzing the daily pattern of consumption, projects sequence of data based on the frequent prefix and reduces the time consumption and the memory heightening the prediction performance. The LSTM learns the error rate occurred in predicting from the actual type and improves the prediction. In future the paper is planned to proceed taking into consideration the factors affecting the pattern of consumption to make the forecasting more real.

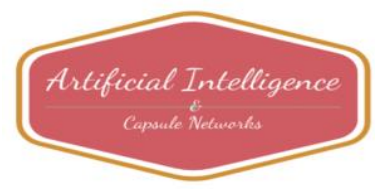


Journal of Artificial Intelligence and Capsule Networks (2020)

Vol.02/ No. 02

Pages: 111-120

http://irojournals.com/aicn/

DOI: https://doi.org/10.36548/jaicn.2020.2.004

\section{References}

[1] Gan, Christopher, V. Limsombunchao, Michael D. Clemes, and Yong YA Weng. "Consumer choice prediction: Artificial neural networks versus logistic models." (2005).

[2] Afolabi, Ibukun T., Olufunke Oladipupo, Rowland E. Worlu, and I. O. Akinyemi. "A systematic review of consumer behaviour prediction studies." Covenant Journal of Business and Social Sciences 7, no. 1 (2016).

[3] Valecha, Harsh, Aparna Varma, Ishita Khare, Aakash Sachdeva, and Mukta Goyal. "Prediction of Consumer Behaviour using Random Forest Algorithm." In 2018 5th IEEE Uttar Pradesh Section International Conference on Electrical, Electronics and Computer Engineering (UPCON), pp. 1-6. IEEE, 2018.

[4] Krebs, Florian, Bruno Lubascher, Tobias Moers, Pieter Schaap, and Gerasimos Spanakis. "Social emotion mining techniques for Facebook posts reaction prediction." arXiv preprint arXiv:1712.03249 (2017).

[5] Urso, Alfonso, Antonino Fiannaca, Massimo La Rosa, Valentina Ravì, and Riccardo Rizzo. "Data mining: Classification and prediction." Encyclopedia of Bioinformatics and Computational Biology: ABC of Bioinformatics (2018): 384.

[6] Sherstinsky, Alex. "Fundamentals of recurrent neural network (rnn) and long short-term memory (lstm) network." arXiv preprint arXiv:1808.03314 (2018).

[7] Staudemeyer, Ralf C., and Eric Rothstein Morris. "Understanding LSTM--a tutorial into Long Short-Term Memory Recurrent Neural Networks." arXiv preprint arXiv:1909.09586 (2019).

[8] Qing, Xiangyun, and Yugang Niu. "Hourly day-ahead solar irradiance prediction using weather forecasts by LSTM." Energy 148 (2018): 461-468.

[9] Mane, Rashmi V., and V. R. Ghorpade. "Predicting student admission decisions by association rule mining with pattern growth approach." In 2016 International Conference on Electrical, Electronics, Communication, Computer and Optimization Techniques (ICEECCOT), pp. 202207. IEEE, 2016.

[10] Raj, Jennifer S. "A Comprehensive Survey on the Computational Intelligence Techniques and Its Applications." Journal of ISMAC 1, no. 03 (2019): 147-159.

[11] Joseph, S. Iwin Thanakumar, and Iwin Thanakumar. "Survey of data mining algorithm's for intelligent computing system." Journal of trends in Computer Science and Smart technology (TCSST) 1, no. 01 (2019): 14-24.

[12] Raj, Jennifer S., and J. Vijitha Ananthi. "Recurrent Neural Networks and Nonlinear Prediction in Support Vector Machines." Journal of Soft Computing Paradigm (JSCP) 1, no. 01 (2019): 33-40.

[13] Bashar, Abul. "Survey on Evolving Deep Learning Neural Network Architectures." Journal of Artificial Intelligence 1, no. 02 (2019): 73-82.

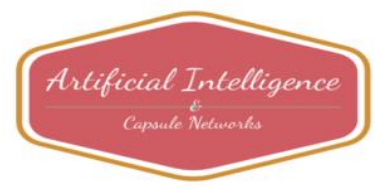


Journal of Artificial Intelligence and Capsule Networks (2020)

Vol.02/ No. 02

Pages: 111-120

http://irojournals.com/aicn/

DOI: https://doi.org/10.36548/jaicn.2020.2.004

\section{Authors Biography}

Dr. P. Karuppusamy working as a Professor and Head in Department of Electrical and Electronics Engineering at Shree Venkateshwara Hi-Tech Engineering College, Erode, India. In 2017, He had completed doctorate in Anna University, Chennai and in 2007, he had completed his post graduate Power Electronics and Drives in Government College of Technology, Coimbatore, India. He has more than 12 years of teaching experience. He has published more than 60 papers in national and international journals and conferences. He has acted as conference chair in IEEE and Springer international conferences and Guest editor in reputed journals. His research area includes Modeling of PV arrays, Adaptive Neuro-Fuzzy Model for Grid Connected Photovoltaic System with Multilevel Inverter. 